\title{
Complex segregation analysis of dilated cardiomyopathy (DCM) in Irish wolfhounds
}

\author{
O Distl ${ }^{1}$, AC Vollmar ${ }^{2}$, C Broschk ${ }^{1}$, H Hamann $^{1}$ and PR Fox ${ }^{3}$ \\ ${ }^{1}$ Institute for Animal Breeding and Genetics, University of Veterinary Medicine Hannover, Hannover, Germany; ${ }^{2}$ Veterinary Clinic for \\ Small Animals, Wissen, Germany and ${ }^{3}$ The Animal Medical Center, New York, NY, USA
}

\begin{abstract}
The objective of the present study was to analyse the mode of inheritance for dilated cardiomyopathy (DCM) in Irish wolfhounds using regressive logistic models by testing for mechanisms of genetic transmission. Insights from this spontaneous animal model should aid importantly in understanding basic pathogenic mechanisms with regard to genetics and molecular biology of DCM in humans. Moreover, a procedure for the simultaneous prediction of breeding values and the estimation of genotype probabilities for DCM is expected to markedly improve breeding programmes. Results of cardiovascular examinations of 1018 dogs carried out between 1987 and 2003 by one veterinarian were analysed. Data of 878 dogs from 531 litters in 147 different
\end{abstract}

kennels were used for complex segregation analyses. Pedigree information was available for more than 15 generations. Male dogs were affected significantly more often by DCM than female dogs. The segregation analysis showed that among all other tested models a mixed monogenic-polygenic model including a sex-dependent allele effect best explained the segregation of affected animals in the pedigrees. A pure monogenic inheritance of DCM could be significantly rejected in favour of the major gene and most general model. The gene action of the major gene was significantly different between female and male dogs.

Heredity (2007) 99, 460-465; doi:10.1038/sj.hdy.6801024; published online 4 July 2007

Keywords: dog; dilated cardiomyopathy; segregation analysis; major gene

\section{Introduction}

Dilated cardiomyopathy (DCM), a primary form of heart muscle disease characterized by impaired myocardial contractility and cardiac chamber dilation, is the most common cause of heart diseases in large and giant dog breeds. Clinical manifestations generally include congestive heart failure and in some animals, sudden cardiac death. An increased prevalence of DCM has been reported in certain canine breeds including pinschers, boxers, Scottish deerhounds, Irish wolfhounds, Great Danes, Saint Bernards, Afghan hounds, Newfoundlands, Old English sheepdogs, English and American cocker spaniels, Dalmatians, Portuguese water dogs, Presa Canario dogs, Labrador retrievers and Golden retrievers (Calvert et al., 1982; Gooding et al., 1986; Harpster, 1991; Sisson and Thomas, 1995; Freeman et al., 1996; Kittleson, 1998; Dambach et al., 1999; Sisson et al., 1999; Vollmar, 2000; Morales et al., 2001; Tidholm et al., 2001). Characteristically, clinical signs of DCM are manifested between 4 and 10 years of age, although juvenile forms leading to rapid death are occasionally recognized (Dambach et al., 1999; Sleeper et al., 2002; Vollmar et al., 2003). Males appear to be more commonly affected than females (Calvert et al., 1982; Harpster, 1991; Everett et al., 1999; Vollmar, 2000).

Correspondence: Professor O Distl, Institute of Animal Breeding and Genetics, University of Veterinary Medicine Hannover, Buenteweg 17p, Hannover 30559, Germany.

E-mail: ottmar.distl@tiho-hannover.de

Received 20 May 2006; revised 10 May 2007; accepted 28 May 2007; published online 4 July 2007
Despite a number of reports regarding inherited aspects of DCM in several canine breeds, the genetic basis underlying this disease in dogs has not been clarified. A heritable basis for DCM has been suggested based upon its increased incidence in specific dog breeds, as well as in certain families within breeds. Autosomal modes of inheritance for DCM were suggested for the Irish wolfhound, Newfoundland and Portuguese water dog (Cobb et al., 1996; Dambach et al., 1999; Dukes-McEwan and Jackson, 2002; Sleeper et al., 2002). DCM was suspected to be an X-linked monogenic recessive trait in the Great Dane breed in one report (Meurs et al., 2001b), while other investigators reported that pedigrees of Newfoundland dogs were most consistent with an incomplete penetrant autosomal monogenic dominant mode of inheritance (Dukes-McEwan and Jackson, 2002). However, the large number of common ancestors made it difficult to conclusively exclude recessive monogenic inheritance. In Portuguese water dog puppies with DCM, the segregation ratios of affected animals did not differ significantly from autosomal monogenic recessive inheritance. Dominant inheritance also allowing for incomplete penetrance was unlikely (Dambach et al., 1999; Alroy et al., 2000). Moreover, the pedigrees analyzed were not sufficiently informative to be able to reject a polygenic model (Alroy et al., 2000). In German short-haired pointers with skeletal myopathy and cardiomyopathy, deletion of the entire dystrophin gene was demonstrated (Schatzberg et al., 1999). Mutations of the actin gene cause a heritable form of DCM in humans, but molecular genetic analyses in Dobermann pinschers with DCM failed to reveal any abnormalities of this gene (Meurs et al., 
2001a). Myocardial samples from Dobermann pinscher, Dalmatian, Bull mastiff and Irish wolfhound breeds were evaluated for abnormalities in dystrophin, $\alpha$ sarcoglycan and $\beta$-dystroglycan proteins using western blot analysis. Differences between the bands of the western blot analysis could not be identified when dogs with DCM were compared with the control group (dogs without myocardial disease). Therefore, abnormalities in these three cytoskeletal proteins are unlikely to be associated with DCM in the dogs investigated (Spier et al., 2001). A study of 48 Newfoundland dogs using a genome-wide scan with over 200 microsatellite markers failed to detect linkage with DCM (Dukes-McEwan and Jackson, 2002).

Dilated cardiomyopathy represents a major cause of congestive heart failure in man, affecting 36.5 per 100000 individuals. The mortality rate of individuals with symptomatic DCM is high, approaching $50 \%$ at 5 years (Hughes and McKenna, 2005). Pedigree analysis using relatives of index patients has been shown that up to $35 \%$ of individuals with DCM have familial disease (Grunig et al., 1998). Patterns of inheritance include autosomal, Xlinked and mitochondrial forms. Inherited DCM is most commonly transmitted as an autosomal dominant trait (Ahmad et al., 2005). The limited availability of large families for linkage studies, the late onset and incomplete penetrance of this disease slow down deciphering of the genetic causes of DCM in man. Dog models can be useful in this respect as large pedigrees are available, indicating similar clinical and pathohistological signs as in humans. The general, much shorter lifespan of dogs (by about a factor of seven) in comparison to man allows for establishing models in reasonable time. In addition, there are dog breeds exhibiting high prevalence of DCM. An important step towards a dog model can be made showing the familiarity and mode of inheritance of canine DCM. Molecular genetic studies in man unravelled 25 different loci causing DCM (Ahmad et al., 2005). In addition, many more DCM regions have been mapped for which the gene remains unknown (Ahmad et al., 2005). This considerable genetic heterogeneity suggested that there are multiple pathways leading to DCM. A dog model appears well suited for delineating molecular and cell events involved in these pathways that lead to heart failure.

The objective of the present study was to analyse the mode of inheritance of the prevalence of DCM in Irish wolfhound dogs by employing complex segregation analyses. Detection and characterization of major genes segregating in DCM of Irish wolfhounds would help investigate complex clinical and pathogenic mechanisms responsible for sudden death and disease progression in human myocardial diseases. In addition, improvements in molecular genetic analysis would help reduce the genomic prevalence of DCM through management of canine breeding programmes.

\section{Materials and methods}

Cardiovascular examinations were performed on 1018 Irish wolfhounds (442 males and 576 females) by one veterinary cardiologist between January 1987 and November 2003. These dogs were 7.6 months to 10.8 years of age with a mean of $3.6 \pm 1.9$ years. Using standardized protocols, for each dog, thoracic auscultation, standard six-lead electrocardiograms and echocardiographic examinations including colour flow Doppler echocardiography were performed. Two-dimensional and M-mode echocardiograms were recorded and analysed according to the recommendations of the American Society of Echocardiography and the Echocardiographic Committee of the Specialty of Cardiology, American College of Veterinary Internal Medicine, CO (Thomas et al., 1993; Kienle and Thomas, 1995). We used reference values reported for the Irish wolfhound breed to distinguish between dogs with DCM and unaffected dogs (Vollmar, $1999 a, b)$. In the present study cardiac phenotype was judged to be unaffected by DCM based upon the most current examination, or affected by DCM based upon the first examination when this diagnosis was made. Data from a total of 878 Irish wolfhounds were used for the genetic evaluations. These 376 male and 502 female probands descended from 220 sires and 413 dams bred in 147 kennels. For construction of pedigrees additional Irish wolfhounds were included, even if the phenotype had not been recorded. At least the parents had to be known for each examined dog. Up to 15 generations were available for some animals. In total, the dataset included 2203 animals. Phenotypes were classified as having normal heart function or DCM. Further information on sex, date of birth and pedigree was recorded by the kennel clubs and collated for this analysis. The inbreeding coefficient was calculated from the pedigrees using all available generations.

The mode of inheritance was tested with class A regressive logistic models (Bonney, 1986; SAGE, Release 3.0). As the pedigrees were randomly sampled, no ascertainment correction was applied. DCM was used as a dichotomous-dependent variable, with normal heart function encoded 0 and DCM 1 . All results obtained by the different models were validated by the use of a set of starting values of all estimated parameters employing a complete grid search for initial values. As DCM is a lateonset disease and the age at last examination or the age at examination when DCM was diagnosed proved significant, all models included age at examination as linear covariate. Different models were employed for testing hypotheses on the mode of inheritance (Table 1). The likelihood of the data was modelled as a function of parameters. These parameters included the probability that each individual could be of type $A A, A B$ or $B B$, whereby each type was associated with a probability for trait expression (equals penetrance in a genetic model); types were modelled using probabilities for transmitting an allele $\mathrm{A}$ to a offspring with types $\mathrm{AA}, \mathrm{AB}$ or $\mathrm{BB}$; residual familial effects were allowed to account for polygenic variation explained through parent-offspring and sib-sib correlations; covariates were used to explain further non-genetic effects. An autosomal locus with two alleles (A and B) was assumed when the model allowed for a possible monogenic factor or a major gene. Genotypes frequencies were estimated assuming Hardy-Weinberg equilibrium. Instead of assuming genetic transmission and genotypes, individuals can be associated with types to account for non-genetic mechanisms (Go et al., 1978). The regressive familial effects were modelled in the logistic regressions of the DCM status of offspring on the phenotype of sire and dam. The effect of the mating partner may explain a possible correlation among mating partners. Therefore, the phenotype of one 
Table 1 Models used in complex segregation analyses for DCM in Irish wolfhound dogs

\begin{tabular}{|c|c|c|}
\hline Hypothesis tested & Genetic effects & Explanation \\
\hline \multicolumn{3}{|c|}{ Phenotypic distributions without considering genetic effects } \\
\hline$\mu$ & No & Only one phenotypic distribution $(\mu)$ \\
\hline$\mu_{\text {male }} / \mu_{\text {female }}$ & No & Two phenotypic distributions for male and female dogs \\
\hline$\mu_{\text {male }} / \mu_{\text {female }}+$ age & & $\begin{array}{l}\text { Two phenotypic distributions for male and female dogs and age as } \\
\text { linear covariate }\end{array}$ \\
\hline$\mu_{\text {male }} / \mu_{\text {female }}+$ age + covariates & No & $\begin{array}{l}\text { Two phenotypic distributions for male and female dogs, age as } \\
\text { linear covariate and covariates for birth year, birth month, country } \\
\text { where the kennel club is registered and inbreeding coefficient }\end{array}$ \\
\hline
\end{tabular}

$\begin{array}{lc}\text { One locus model (autosomal biallelic) }+\mu_{\text {male }} / \mu_{\text {female }}+\text { covariates }+ \text { age } \\ \text { Recessive } & \text { Monogenic } \\ \text { Dominant } & \text { Monogenic } \\ \text { Arbitrary } & \text { Monogenic } \\ & \\ \text { Polygenic model }+\mu_{\text {male }} / \mu_{\text {female }}+\text { covariates } & \text { age } \\ \text { Type } 1 & \text { Polygenic } \\ \text { Type } 3 & \text { Polygenic } \\ \text { Type } 5 & \text { Polygenic } \\ \text { Type } 7 & \text { Polygenic }\end{array}$

Mixed monogenic-polygenic model $+\mu_{\text {male }} / \mu_{\text {female }}+$ covariates + age Arbitrary - types 1, 3, $5 \quad$ Monogenic+polygenic

Recessive - type 7

Dominant - type 7

Arbitrary - type 7

Monogenic+polygenic

Monogenic+polygenic

Monogenic+polygenic
Sex-dependent, recessive allele effect

Sex-dependent, dominant allele effect

Sex-dependent, arbitrary allele effect

Equal polygenic effects of affected parents

Polygenic effects of affected sires and dams

Polygenic effects of unaffected and affected parents

Polygenic effects of unaffected and affected sires and dams

Sex-dependent, arbitrary major gene effect and polygenic effects of parents (type 1) or affected sire and dams (type 3) or unaffected and affected parents (type 5)

Sex-dependent, recessive major gene effect and polygenic effects of unaffected and affected sires and dams

Sex-dependent, dominant major gene effect and polygenic effects of unaffected and affected sires and dams

Sex-dependent, arbitrary major gene effect and polygenic effects of unaffected and affected sires and dams specific mating partner of each full-sib family was always regressed on the phenotype of the other mating partner. We had to include the mating partner correlation in our analysis as there were mating loops in the pedigrees that would have been omitted otherwise.

The basic logistic model in general terms was fitted to the data as follows:

$$
\vartheta=\alpha\left(u_{\mathrm{i}}\right)+\delta_{\mathrm{F}} Z_{\mathrm{F}}+\delta_{\mathrm{M}} Z_{\mathrm{M}}+\delta_{\mathrm{S}} Z_{\mathrm{S}}+b_{\mathrm{i}} X_{\mathrm{i}}
$$

where $\vartheta$, is the observed status for DCM with 0 for normal heart function and 1 for DCM, $\alpha$, the baseline parameter, defined according to model used for $u_{\mathrm{i}}$ (monogenic inheritance or no monogenic inheritance), $\delta_{\mathrm{F}, \mathrm{M}, \mathrm{S}}$, the logistic regression coefficients for sire $(\mathrm{F})$, dam $(\mathrm{M})$ or mating partner (S), $b_{\mathrm{i}}$, the logistic regression coefficients for covariates: age, birth year, birth month, country where the kennel club is registered, inbreeding coefficient, $Z_{\mathrm{F}, \mathrm{M}, \mathrm{S}}$, the explanatory variables for the design matrix using observed values for the phenotypic trait (DCM) of sire (F), dam (M) or mating partner (S) and $X_{\mathrm{i}}$, the explanatory variables for the covariates age, birth year, birth month, country where the kennel club is registered, inbreeding coefficient.

Likelihood ratio tests were used to evaluate the goodness of fit of the model to the data. Therefore, a saturated model was defined with no restrictions on parameters in the model used. The likelihood ratio test statistic compares a specific null hypothesis $\left(H_{0}\right)$ defined by a restricted model against a saturated (most general) model. The test statistic is given by the difference of the $\log$ likelihoods multiplied by minus two. The ratio of $\log$ likelihoods asymptotically follows a $\chi^{2}$-distribution, and significance levels can be obtained by using this distribution. Degrees of freedom are given by the difference of independently estimated parameters for the models compared. The information criterion of Akaike (AIC) was used as an additional measure for choosing the sparsest model with the best fit to the data. AIC is given by the log likelihood multiplied by minus two plus two times the number of independently estimated parameters. The model with the smallest AIC fits the data best with a minimum number of parameters but all hypotheses that cannot be rejected against the most general model using the likelihood ratio test must also be considered as possible. The AIC criterion can be used to differentiate between alternative models with different numbers of parameters to be estimated if more than one hypothesis that was tested against the most general model is accepted. Nevertheless, the AIC criterion cannot be used to rule out a hypothesis if this model was not rejected in favour of the most general model by using the likelihood ratio test.

\section{Results}

Out of the 1018 dogs, at the time of presentation DCM was diagnosed in 260 dogs (25.5\%; 148 males and 112 females) resulting in a prevalence of DCM in $33.5 \%$ of all male dogs and $19.4 \%$ of all females. The mean age at the time of diagnosis of DCM was $4.40 \pm 2.03$ years, with a range from 7.6 months to 10.8 years of age. The mean age of dogs classified as unaffected was $3.3 \pm 1.7$ years with a range from 10.1 months to 9.7 years. 
The mean of the inbreeding coefficient was $4.26 \%$, and a maximum of $23.4 \%$. Among all dogs examined, $25.9 \%$ were affected by DCM, $34.0 \%$ of the male and $19.7 \%$ of

Table 2 Distribution (\%) of Irish wolfhounds $(n=878)$ by dilated cardiomyopathy and sex, birth year, birth month and inbreeding coefficient

\begin{tabular}{|c|c|c|c|}
\hline Source of variation & $\mathrm{n}$ & Unaffected by DCM & $D C M$ \\
\hline Male & 376 & 66.0 & 34.0 \\
\hline Female & 502 & 80.3 & 19.7 \\
\hline \multicolumn{4}{|l|}{ Birth year } \\
\hline$<1994$ & 205 & 57.6 & 42.4 \\
\hline 1994-1995 & 173 & 72.3 & 27.7 \\
\hline 1996-1997 & 265 & 76.6 & 23.4 \\
\hline 1998-2001 & 235 & 87.2 & 12.8 \\
\hline \multicolumn{4}{|l|}{ Birth month } \\
\hline Jan-Mar & 230 & 74.8 & 25.2 \\
\hline Apr-Jun & 226 & 69.9 & 30.1 \\
\hline Jul-Sep & 232 & 75.4 & 24.6 \\
\hline Oct-Dec & 190 & 79.0 & 21.0 \\
\hline \multicolumn{4}{|c|}{ Country of origin of the kennel club } \\
\hline Germany & 277 & 67.9 & 32.1 \\
\hline Netherlands & 236 & 76.3 & 23.7 \\
\hline Belgium & 118 & 76.3 & 23.7 \\
\hline Other countries & 247 & 78.1 & 21.9 \\
\hline \multicolumn{4}{|c|}{ Inbreeding coefficient (\%) } \\
\hline 0 & 177 & 77.4 & 22.6 \\
\hline$>0-2.5$ & 270 & 75.2 & 24.8 \\
\hline$>2.5-5.0$ & 131 & 68.7 & 31.3 \\
\hline$>5.0-7.5$ & 109 & 76.2 & 23.8 \\
\hline$>7.5-10.0$ & 85 & 81.2 & 18.8 \\
\hline$>10.0-12.5$ & 28 & 67.9 & 32.2 \\
\hline$>12.5$ & 78 & 64.1 & 35.9 \\
\hline
\end{tabular}

Abbreviation: DCM, dilated cardiomyopathy. the female dogs. This difference between male and female gender was statistically significant at $P<0.001$. Table 2 contains the distribution of DCM by sex, classes of birth years and birth months, country of the kennel club, and inbreeding coefficient.

The results of the complex segregation analysis are shown in Table 3. These models considered the effects of sex and age on DCM. In addition, we tested models with further phenotypic distributions including birth year, birth month, country where the kennel club was registered and inbreeding coefficient (Table 4). The hypotheses on the mode of inheritance included monogenic, polygenic and mixed monogenic-polygenic inheritance. The likelihood ratio test statistic significantly rejected models accounting only for one or more phenotypic distribution. A sex-dependent effect was significant and thus was included in all further models used. A major gene model including a sex-dependent genotype effect fitted the data significantly better than all other models employed. Models with arbitrary major gene effects always resulted in estimates that explained a significantly larger proportion of variances than models with recessive or dominant major gene effects. Also the contributions of affected and not-affected dams and sires differed significantly and the inclusion of these familiar effects improved the model substantially. This result could be confirmed under different parameterizations with covariates. The $-2 \log$ likelihood was smallest for the major gene model with familial effects option 7 and arbitrary gene action in the models extended by the covariates birth year, birth month and country of origin or inbreeding coefficient (Table 4). The AIC was also lowest for this mixed monogenic-polygenic model. Therefore, this model gave the best possible explanation of the segregation of DCM in this random sample of 878 Irish wolfhounds investigated.

Table 3 Complex segregation analysis using regressive logistic models for occurrence of DCM in Irish wolfhound dogs

\begin{tabular}{|c|c|c|c|c|c|c|c|}
\hline Hypothesis tested & Monogenic transmission & Familial effects & $-2 \ln L$ & AIC & d.f. & $\chi^{2}$ & $\mathrm{P}$ \\
\hline$\mu+$ age & No & No & 1122.1 & 1126.1 & 15 & 96.90 & $<0.001$ \\
\hline$\mu_{\text {male }} / \mu_{\text {female }}+$ age & No & No & 1089.0 & 1095.0 & 14 & 63.70 & $<0.001$ \\
\hline \multicolumn{8}{|c|}{ One locus model $+\mu_{\text {male }} / \mu_{\text {female }}+$ age } \\
\hline Recessive & Yes & No & 1082.1 & 1094.1 & 11 & 56.70 & $<0.001$ \\
\hline Dominant & Yes & No & 1055.3 & 1067.3 & 11 & 29.80 & 0.001 \\
\hline Arbitrary & Yes & No & 1054.6 & 1070.6 & 9 & 29.0 & $<0.01$ \\
\hline \multicolumn{8}{|c|}{ Polygenic model $+\mu_{\text {male }} / \mu_{\text {female }}+$ age } \\
\hline Type 1 & No & Yes & 1066.6 & 1076.6 & 12 & 40.90 & $<0.001$ \\
\hline Type 3 & No & Yes & 1057.1 & 1069.1 & 11 & 31.30 & $<0.001$ \\
\hline Type 5 & No & Yes & 1064.9 & 1078.9 & 10 & 31.0 & 0.015 \\
\hline Type 7 & No & Yes & 1044.1 & 1062.1 & 8 & 19.0 & $<0.001$ \\
\hline \multicolumn{8}{|c|}{ Mixed monogenic-polygenic model $+\mu_{\text {male }} / \mu_{\text {female }}+$ age } \\
\hline Type 1 - recessive & Yes & Yes & 1062.1 & 1078.1 & 9 & 36.99 & 0.007 \\
\hline Type 1 - dominant & Yes & Yes & 1047.7 & 1063.1 & 9 & 22.58 & 0.020 \\
\hline Type 1 - arbitrary & Yes & Yes & 1041.7 & 1061.7 & 7 & 16.57 & 0.030 \\
\hline Type 3 - arbitrary & Yes & Yes & 1039.1 & 1061.1 & 6 & 13.96 & 0.009 \\
\hline Type 5 - arbitrary & Yes & Yes & 1040.4 & 1064.4 & 5 & 15.25 & 0.008 \\
\hline Type 7 - recessive & Yes & Yes & 1040.9 & 1064.9 & 5 & 15.74 & 0.008 \\
\hline Type 7 -dominant & Yes & Yes & 1034.8 & 1058.8 & 5 & 9.63 & 0.086 \\
\hline Type 7 - arbitrary & Yes & Yes & 1028.0 & 1056.0 & 3 & 2.82 & 0.420 \\
\hline Saturated model & No & Yes & 1025.2 & - & - & - & - \\
\hline
\end{tabular}

Abbreviations: AIC, information criterion according to Akaike; d.f., degrees of freedom; $\ln \mathrm{L}, \log$ likelihood.

$\chi^{2}$ compares the model tested with the most general model. 
Table 4 Complex segregation analysis using regressive logistic models and including additional phenotypic distributions in the model for occurrence of DCM in Irish wolfhound dogs

\begin{tabular}{|c|c|c|c|c|c|c|c|}
\hline Hypothesis tested & Monogenic transmission & Familial effects & $-2 \ln L$ & AIC & d.f. & $\chi^{2}$ & $\mathrm{P}$ \\
\hline$\mu_{\text {male }} / \mu_{\text {female }}+$ age & No & No & 1089.0 & 1095.0 & 25 & 91.30 & $<0.001$ \\
\hline$\mu_{\text {sex }}+\mu_{\text {covariates }}+$ age & No & No & 1053.3 & 1077.3 & 15 & 55.50 & $<0.001$ \\
\hline \multicolumn{8}{|c|}{ One locus model $+\mu_{\text {sex }}+\mu_{\text {covariates }}+$ age } \\
\hline Recessive & Yes & No & 1040.6 & 1070.6 & 12 & 42.70 & $<0.001$ \\
\hline Dominant & Yes & No & 1023.2 & 1053.2 & 12 & 26.10 & 0.010 \\
\hline Arbitrary & Yes & No & 1019.6 & 1053.6 & 10 & 22.49 & 0.013 \\
\hline \multicolumn{8}{|c|}{ Polygenic model $+\mu_{\text {sex }}+\mu_{\text {covariates }}+$ age } \\
\hline Type 1 & No & Yes & 1038.8 & 1066.8 & 13 & 41.68 & $<0.001$ \\
\hline Type 3 & No & Yes & 1026.4 & 1056.4 & 12 & 29.27 & 0.004 \\
\hline Type 5 & No & Yes & 1037.4 & 1069.4 & 11 & 40.26 & $<0.001$ \\
\hline Type 7 & No & Yes & 1013.7 & 1049.7 & 9 & 16.55 & 0.056 \\
\hline \multicolumn{8}{|c|}{ Mixed monogenic-polygenic model $+\mu_{\text {sex }}+\mu_{\text {covariates }}+$ age } \\
\hline Type 1 - arbitrary & Yes & Yes & 1011.1 & 1049.1 & 8 & 13.94 & 0.083 \\
\hline Type 3 - arbitrary & Yes & Yes & 1008.9 & 1048.9 & 7 & 11.73 & 0.110 \\
\hline Type 5 - arbitrary & Yes & Yes & 1009.9 & 1051.9 & 6 & 12.72 & 0.048 \\
\hline Type 7 - recessive & Yes & Yes & 1012.5 & 1054.5 & 6 & 15.31 & 0.018 \\
\hline Type 7 - dominant & Yes & Yes & 1006.3 & 1048.3 & 6 & 9.10 & 0.168 \\
\hline Type 7 - arbitrary & Yes & Yes & 999.5 & 1045.5 & 4 & 2.29 & 0.683 \\
\hline Saturated model & No & Yes & 997.7 & - & - & - & \\
\hline
\end{tabular}

Abbreviations: AIC, information criterion according to Akaike; d.f., degrees of freedom; ln L, $\log$ likelihood.

$\chi^{2}$ compares the model tested with the most general model.

\section{Discussion}

A sex-dependent major gene model with arbitrary gene action and specific effects of affected and not-affected dams and sires gave the best fit to the pedigrees collected. Thus, development of DCM can be attributed to a single gene locus and further polygenic effects. This single gene plays a major role in the expression of DCM, but alone cannot sufficiently explain the occurrence of this disease.

The significance of the polygenic component in our study indicates that there might be additional gene loci that contribute to the development of DCM. It is not possible to determine the number of loci involved using regressive logistic models. The genetic model found most likely in our analysis conclusively confirmed the genetic basis of late-onset DCM suggested by different authors (Cobb et al., 1996; Schatzberg et al., 1999; Meurs et al., 2001b; Dukes-McEwan and Jackson, 2002). In fact, the finding of a major gene presented here is the first report that could find evidence for the mode of inheritance of DCM in Irish wolfhounds. The robustness of these results was not affected by the inclusion of additional covariates such as birth year, birth month, country of origin and inbreeding coefficient. In accordance with previous reports, we found a higher prevalence of DCM in male dogs (Calvert et al., 1982; Harpster, 1991; Everett et al., 1999; Vollmar, 2000). Furthermore, our analysis showed different genotypic distributions among male and female dogs. This finding may indicate that genes may act differently among sexes or possibly that Xlinked genes may have some minor importance for expression of DCM.

Detection of major genes in large pedigrees can greatly increase the power of genome-wide scans or candidate gene-associated marker studies, to establish a discordant sib study design. Family members identified as carriers of the major gene can be preferentially genotyped in such studies and non-carriers can be used as controls. A discordant sib design proved as powerful tool for detecting a candidate gene or a linked marker (Risch and Zhang, 1995). In man over 25 different loci have been identified by linkage analysis or candidate screening strategies as being responsible for familial DCM (DukesMcEwan and Jackson, 2002). Gene mutations corresponding to these loci have been identified in the laminin $\mathrm{A} / \mathrm{C}-$, troponin $\mathrm{T}-$, titin-, $\beta$-myosin heavy chain-, cysteine- and -glycine-rich protein 3-, phospholamban-, cardiac myosin-binding protein $\mathrm{C}-$, telethonin-, dystrophin-, tafazzin-, isoleucine tRNA-, cardiac actin-, desmin-, $\alpha$-tropomyosin- and cardiac $\delta$-sarcoglycan gene. Additional candidate genes provide mouse and hamster models. DCM genes detected in mice are the genes encoding desmin, cAMP response element-binding protein, cathepsin L and MARCKS-like protein. DCM in man appears genetically heterogeneous. Our results are not contradictory as in dogs DCM may also not be genetically homogeneous. However, proof of this hypothesis would be only possible if breeds affected by DCM are crossbred or the mutations of genes responsible for DCM in the different dogs breeds have been identified.

Use of comparative genomics in combination with candidate gene-associated marker scans for specific chromosomal locations and genome-wide scans would allow detection of the molecular basis of the DCM in Irish wolfhound dogs. Characterization of the mutation(s) of the major gene that account for a large proportion of DCM in Irish wolfhounds would make it possible to develop a powerful gene test. In addition, the genetic approach proposed here to unravel the molecular genetic basis of DCM in Irish wolfhounds might become a powerful tool for identifying animal models for complex traits. 


\section{References}

Ahmad F, Seidman JG, Seidman CE (2005). The genetic basis for cardiac remodelling. Annu Rev Hum Genet 6: 185-216.

Alroy J, Rush JE, Freeman L, Amarendhra Kumar MS, Karuri A, Chase K et al. (2000). Inherited infantile dilated cardiomyopathy in dogs: genetic, clinical, biochemical, and morphologic findings. Am J Med Genet 6: 57-66.

Bonney GE (1986). Regressive logistic models for familial disease and other binary traits. Biometrics 42: 611-625.

Calvert CA, Chapman W, Toal RL (1982). Congestive cardiomyopathy in Doberman pinscher dogs. J Am Vet Med Assoc 181: 598-602.

Cobb MA, Brownlie S, Pidduck SE, Batt RM (1996). Evidence for Genetic Involvement in Dilated Cardiomyopathy in the Irish Wolfhound. Ann Congress BSAVA: Birmingham. p 215.

Dambach DM, Lannon A, Sleeper MM, Buchanan J (1999). Familial dilated cardiomyopathy of young Portuguese water dogs. J Vet Intern Med 13: 65-71.

Dukes-McEwan J, Jackson IJ (2002). The promises and problems of linkage analysis by using the current canine genome map. Mamm Genome 13: 667-672.

Everett RM, McGann J, Wimberly HC, Althoff J (1999). Dilated cardiomyopathy of Doberman pinschers: retrospective histomorphologic evaluation of heart from 32 cases. Vet Pathol 36: 221-227.

Freeman LM, Michel KE, Brown DJ, Kaplan PM, Stamoulis ME, Rosenthal SL et al. (1996). Idiopathic dilated cardiomyopathy in Dalmatians: nine cases (1990-1995). J Am Vet Med Assoc 209: 1592-1596.

Go RC, Elston RC, Kaplan EB (1978). Efficiency and robustness of pedigree segregation analysis. Am J Hum Genet 30: 28-37.

Gooding JP, Robinson WF, Mews GC (1986). Echocardiographic characterization of dilatation cardiomyopathy in the English Cocker Spaniel. Am J Vet Res 47: 1978-1983.

Grunig E, Tasman JA, Kucherer A, Franz W, Kubler W, Katus HA (1998). Frequency and phenotypes of familial dilated cardiomyopathy. J Am Coll Cardiol 31: 186-194.

Harpster NK (1991). Boxer cardiomyopathy. A review of longterm benefits of antiarrhythmic therapy. Vet Clin North Am Small Anim Pract 21: 989-1004.

Hughes SE, McKenna WJ (2005). New insights into pathology of inherited cardiomyopathy. Heart 91: 257-264.

Kienle RD, Thomas WP (1995). Echocardiography, recommended methods for echocardiographic measurements. In: Nyland TG, Mattoon JS (eds). Veterinary Diagnostic Ultrasound. Saunders: Philadelphia. pp 206-208.

Kittleson M (1998). Primary myocardial disease leading to chronic myocardial failure (dilated cardiomyopathy and related diseases). In: Kittleson M, Kienle RD (eds). Small Animal Cardiovascular Medicine. Mosby: St Louis. pp 319-346.
Meurs KM, Magnon AL, Spier AW, Miller MW, Lehmkuhl LB, Towbin JA (2001a). Evaluation of the cardiac actin gene in Dobermann pinschers with dilated cardiomyopathy. I Am Vet Res 62: 33-36.

Meurs KM, Miller MW, Wright NA (2001b). Clinical features of dilated cardiomyopathy in Great Danes and results of a pedigree analysis: 17 cases (1999-2000). J Am Vet Med Assoc 218: 729-732.

Morales M, Ynaraja E, Montoya JA (2001). Dilated cardiomyopathy in Presa Canario dogs: ECG findings. J Vet Med 48: 577-580.

Risch N, Zhang H (1995). Extreme discordant sib pairs for mapping quantitative trait loci in humans. Science $\mathbf{2 6 8}$ 1584-1589.

Schatzberg SJ, Olby NJ, Breen M, Anderson LV, Langford CF, Dickens HF et al. (1999). Molecular analysis of a spontaneous dystrophin 'knockout' dog. Neuromuscul Disord 9: 289-295.

Sleeper MM, Henthorn PS, Vijayasarathy C, Dambach DM, Bowers T, Tijskens P et al. (2002). Dilated cardiomyopathy in juvenile Portuguese water dogs. J Vet Intern Med 16: 52-62.

Spier AW, Meurs KM, Coovert DD, Lehmkuhl LB, O'grady MR, Freeman LM et al. (2001). Use of western immunoblot for evaluation of myocardial dystrophin, alpha-sarcoglycan, and beta-dystroglycan in dogs with dilated cardiomyopathy. J Am Vet Res 62: 67-71.

Sisson DD, Thomas WP (1995). Myocardial diseases, the prevalence of dilated cardiomyopathy. In: Ettinger SJ, Feldman EC (eds). Textbook of Veterinary Internal Medicine, 4th edn. Saunders: Philadelphia. pp 995.

Sisson DD, O'Grady MR, Calvert CA (1999). Myocardial diseases of dogs. In: Fox PR, Sisson DD, Moise NS (eds). Textbook of Canine and Feline Cardiolooy, 2nd edn. Saunders: Philadelphia. pp 582-585.

Thomas WP, Gaber CE, Jacobs GJ, Kaplan M, Lombard CW, Moise NS et al. (1993). Recommendations for standards in transthoracic two-dimensional echocardiography in the dog and cat. J Vet Int Med 7: 247-252.

Tidholm AJ, Häggström M, Tarducce BA (2001). Canine idiopathic dilated cardiomyopathy. Part I: aetiology, clinical characteristics, epidemiology and pathology. Vet J 162: 92-107.

Vollmar A (1999a). Echocardiographic measurements in Irish wolfhounds, reference values for the breed. J Am Anim Hosp Assoc 35: 271-277.

Vollmar A (1999b). Use of echocardiography in the diagnosis of dilated cardiomyopathy in Irish wolfhounds. J Am Anim Hosp Assoc 35: 279-283.

Vollmar A (2000). The prevalence of cardiomyopathy in the Irish wolfhound: a clinical study of 500 dogs. J Am Anim Hosp Assoc 36: 125-132.

Vollmar A, Fox MR, Meurs KM, Liu SK (2003). Dilated cardiomyopathy in juvenile Doberman pinschers. I Vet Cardiol 5: 23-27. 